\title{
Schweigepflicht: Lieber ein Wort weniger, als zu viel
}

Die ärztliche Schweigepflicht ist nicht nur relevant für

Ärzte, sondern sie gilt auch für Mitarbeiter. Für Medizinische

Fachangestellte kann selbst das Telefonat am Empfang vor

Publikum schnell zur Falle werden.

Der Fall: Am Empfang einer Gemeinschaftspraxis klingelt das Telefon. Die Patienten im Wartezimmer hören neugierig zu, wie die Medizinische Fachangestellte (MFA) den Anrufer mit Namen begrüßt und ihm im Laufe des Telefonats mitteilt, wie sein Befund aussieht.

Aus gutem Grund werden Ärzte durch die Berufsordnungen ihrer Landesärztekammern einer umfassenden Schweigepflicht unterworfen: Die ärztliche Verschwiegenheit ist eine wesentliche Grundlage des Arzt-Patienten-Verhältnisses. Schon die Anamnese kann nur dann in größtmöglichem Umfang erhoben werden, wenn der Patient darauf vertrauen kann, dass seine Angaben allein zu Behandlungszwecken verwendet werden. So schreibt Paragraf 9 Absatz 1 der Musterberufsordnung für Ärzte vor, dass ein Arzt über alles schweigen muss, was ihm in seiner Eigenschaft als Arzt anvertraut oder bekannt geworden ist; und dies sogar über den Tod des Patienten hinaus.

\section{Selbst strafrechtliche Sanktionen sind möglich}

Ausdrücklich unterwirft das Berufsrecht die Aufzeichnungen über Patienten und Befunde der Verschwiegenheitspflicht. Eine Verletzung dieser Schweigepflicht ist ein berufsrechtlicher Verstoß, der ein berufsgerichtliches Verfahren oder sogar ein Approbationsentziehungsverfahren nach sich ziehen kann. Der Gesetzgeber erachtet die ärztliche Verschwiegenheit zudem als so gewichtig, dass er den Bruch der ärztlichen Schweigepflicht strafrechtlich sanktioniert. In Paragraf 203 Absatz 1 Strafgesetzbuch wird für die Verletzung von Privatgeheimnissen durch einen Arzt eine Freiheitsstrafe bis zu einem Jahr oder Geldstrafe angedroht.

Es ist völlig unstreitig, dass auch Befunde sowie die Inhalte der Patientenakte strafrechtlich den Privatgeheimnissen zuzuordnen sind. Damit das auf der ärztli- chen Verschwiegenheit beruhende Vertrauensverhältnis zwischen Arzt und $\mathrm{Pa}$ tient umfassend geschützt wird, ordnen sowohl die Berufsordnungen als auch das Strafrecht an, dass die berufsmäßigen Gehilfen der Ärzte und die Personen in der Ausbildung zu den Hilfsberufen ebenfalls zur Verschwiegenheit verpflichtet sind. Außerdem verlangt das Berufsrecht von Ärzten, dass sie die Einhaltung der Schweigepflicht durch ihre Mitarbeiter überwachen. Deshalb droht bei einer Verletzung der Schweigepflicht durch einen Praxismitarbeiter nicht nur diesem die strafrechtliche Verfolgung, sondern auch dem Praxisinhaber selbst.

\section{Echte Falle im Praxisalltag}

Im Praxisalltag kann das zu einer echten Falle werden, wenn in der Praxis wartende Patienten Telefonate der MFA mithören können. Sobald die Anrufer mit ihrem Namen angesprochen werden, können die Wartenden die ausgetauschten Informationen mit einer Person verbinden. Bereits der Umstand, dass eine Person überhaupt Patient dieser Praxis ist, zählt zu den Geheimnissen, die vom Arzt und infolgedessen auch von den Mitarbeitern zu wahren sind. Die in einem Telefonat übermittelten Befunde oder Laborwerte dürfen natürlich ebensowenig anderen Patienten bekannt werden. Für die MFA sind die Telefongespräche mit Patienten unter dem Gesichtspunkt der Verschwiegenheitsverpflichtung deshalb eine besondere Herausforderung. Sobald Patienten in den Praxisräumen die Telefonate mit anderen Patienten hören können, sollte die namentliche Anrede der Anrufer unterbleiben.

Sicherlich ist es unter Aspekten der Höflichkeit sowie gelingender Kommunikation vorzuziehen, die Patienten auch am Telefon namentlich anzusprechen. Wenn die Telefonanrufe außerhalb der Hörweite anderer Patienten geführt werden können, spricht aus Erwägungen der
Schweigepflicht selbstverständlich nichts dagegen. Gleiches gilt für die telefonische Übermittlung medizinischer Befunde. In diesem Fall ist zusätzlich zu beachten, dass gerade die Befundung von Laborwerten eine ärztliche Leistung ist. Selbst wenn die MFA über genügend Erfahrung verfügen mag, laboratoriumsmedizinische Parameter zu würdigen, muss doch immer der Arzt einen Blick auf die Ergebnisse geworfen haben.

Ohne ärztliche Konsultation kann die Einschätzung eines Laborwertes durch einen nicht-ärztlichen Mitarbeiter nicht nur ein Verstoß gegen die Schweigepflicht sein, sondern auch haftungsrechtliche Risiken bergen. Die Mitarbeiter sind auf die Beachtung der Schweigepflicht also ausdrücklich hinzuweisen.Darüber hinaus sollten klare Regelungen für die telefonische Weitergabe von Befunden getroffen werden. Die Belehrungen der Mitarbeiter sowie der Erlass entsprechender Dienstanweisungen sind zu dokumentieren.

Dies dient einerseits der Beweisführung im Streitfall, andererseits erfüllt der Arzt so eine weitere berufsrechtliche Pflicht. Denn die Berufsordnungen der Landesärztekammern schreiben die Belehrung des Praxispersonals und die schriftliche Dokumentation der Belehrung vor.

Sensible Fragen der Schweigepflicht In unserem oben dargestellten Fall haben die Ärzte der Gemeinschaftspraxis ihren Mitarbeitern im Nachgang noch einmal die sensiblen Fragen der Schweigepflicht auf Grundlage einer von den Mitarbeitern zu unterzeichnenden schriftlichen Erklärung erörtert. Für die am Empfang mit $\mathrm{Pa}$ tienten geführten Gespräche und Telefonate und für die telefonische BefundÜbermittlung gab es zusätzlich schriftliche Dienstanweisungen. (Christian Pinnow)

Ärztezeitung, 19.10.2012, in Zusammenarbeit mit der Berliner Rechtsanwaltskanzlei Dierks und Bohle 